\title{
Corrections to: On Direct Representations of Quasi-injectives and Quasi-projectives
}

\author{
By
}

KENT R. FULLeR

In [2] we wrote $M^{A}\left(M^{(A)}\right)$ for a direct product (sum) of $|A|$ copies of a module $M$. Then we proceeded to make the false assertion that $f \rightarrow g$ where

$$
[g(a)](b)=[f(b)](a), \quad a \in A, b \in B
$$

defines an isomorphism

$$
\left(M^{A}\right)^{(B)} \rightarrow\left(M^{(B)}\right)^{A} .
$$

Since then we have come to realize that this map is in general, merely monic. This error invalidates the proofs given for Theorems 2.3 and 4.2 of that paper. Fortunately, however, the theorems themselves are correct, as can easily be seen from [3, Corollary 2.2] for the latter, and the remark following that corollary or [5, Proposition 3.1] for the former.

Also, we wish to point out that K. A. BYRD has independently proved a version of Theorem 5.1 of [2] that does not require the $\Sigma$ in condition (c). This can be done with a straightforward application of [4, Proposition 1] and [1, Theorem 5.3].

\section{Relerences}

[1] C. FAITH and E. A. WALkER, Direct-sum representations of injective modules. J. Algebra 5, $203-221(1967)$.

[2] K. R. Fuller, On direct representations of quasi-injectives and quasi-projectives. Arch. Math. 20, 495-502 (1969).

[3] K. R. Fuller and D. A. HnL, On quasi-projective modules via relative projectivity. Arch. Math. 21, 369-373 (1970).

[4] É. De Robert, Projectifs et injectifs relatifs. C. R. Acad. Sci. Paris Sér. A-B 286, 361 A -364 A (1969).

[5] C. Trsseron, Quelques propriétés des modules quasi-injectifs. C. R. Acad. Sci. Paris Sér. A-B 268, 1327 A-1380A (1969).

Eingegangen am 16.3.1970

Anschrift des Autors:

Kent R. Fuller

Department of Mathematics

University of Iowa

Iowa City, Iowa 52240, U.S.A. 